\title{
Coartación de la aorta: una revisión sistemática
}

\section{Aorta coartation: a systematic review}

\author{
Ruíz Pérez $0^{1}$, M éndez Duran LR² \\ ${ }^{1}$ Universidad Simón Bolívar (Col), ${ }^{2}$ IPS Universitaria de Antioquía (Camino Universitario distrital) (Col),
}

Resumen - La coartación de la aorta es una patología caracterizada por el estrechamiento de la arteria aorta, con la consecuente obstrucción de su flujo; localizada con mayor frecuencia a nivel de aorta torácica descendente distal al origen de la subclavia izquierda, y en la pared posterior de la arteria. Supone aproximadamente el 5,1\% (3-10\%) de las cardiopatías congénitas y constituye la octava malformación cardiaca por orden de frecuencia. La presentación clínica de la coartación aórtica es muy variada y depende de la severidad de la lesión y de su posible asociación con otras anomalías cardiacas. Se resalta la tipo infantil y tipo adulto, siendo la primera la que reviste mayor morbimortalidad. La ecocardiografía bidimensional y la ecocardiografía doppler son el método diagnóstico fundamental para la coartación aórtica. La angio rresonancia magnética (angio-RM) contrastada, permite hacer una evaluación detallada de la aorta mediante una reconstrucción tridimensional que muestra claramente sus defectos. Por su parte, la TAC multicorte permite la visualización tridimensional del arco desde múltiples planos, pero como inconveniente tiene la emisión de radiación. El cateterismo cardiaco, constituye más que un medio diagnóstico, uno terapéutico en el cual, no sólo se demuestra la localización y extensión de la zona coartada, la presencia de lesiones asociadas y/o de circulación colateral, sino que permite la obtención de un conocimiento fiable de la severidad y repercusión hemodinámica de la misma. El tratamiento de esta patología, puede ser inicialmente médico, pero de forma definitiva por cateterismo cardíaco intervencionista y con menor frecuencia corrección quirúrgica.

Palabras Clave: angiorresonancia magnética, cateterismo cardiaco, ductus arterioso.

Abstract - Coarctation of the aorta is a pathology characterized by narrowing of the aorta, with consequent obstruction of its flow; Located more frequently at the level of the descending thoracic aorta distal to the origin of the left subclavian, and in the posterior wall of the artery. It accounts for approximately $5.1 \%$ (3-10\%) of congenital heart defects and constitutes the eighth cardiac malformation in order of frequency. The clinical presentation of aortic coarctation is very varied and depends on the severity of the lesion and its possible association with other cardiac abnormalities. It emphasizes the type of infant and adult type, the first being the one with the highest morbidity and mortality. Two-dimensional echocardiography and Doppler echocardiography are the fundamental diagnostic method for aortic coarctation. Magnetic resonance angiography (MR) allows a detailed evaluation of the aorta through a three-dimensional reconstruction that clearly shows its defects. On the other hand, the multi-cut TAC allows the three-dimensional visualization of the arc from multiple planes, but as an inconvenience it has the emission of radiation. Cardiac catheterization is more than a diagnostic means, a therapeutic one in which not only the location and extent of the alveolar area, the presence of associated lesions and / or collateral circulation is

Correspondencia: Ornella Ruíz Pérez. Facultad de Ciencias de la Salud - Programa de Pediatría. Universidad simón Bolívar. Cr $59 \# 59$ - 65 El Prado. Barranquilla, Atlántico, Colombia. ornella.ruiz@hotmail.com.

Citar: Ruíz O, Méndez LR. Coartación de la aorta: una revisión sistemática. Cienc. innov. salud. 2015; 3 (2):31 - 42 .

Recibido: Feb. 08 de 2015 / Modificado: May. 15 de 2015 / Aceptado: Jul. 21 de 2015 demonstrated, but also allows the obtaining of a knowledge Reliability of the severity and hemodynamic repercussion of the same. The treatment of this pathology can be initially medical, but definitively by interventional cardiac catheterization and less frequently surgical correction.

Keywords: Magnetic resonance angiography, cardiac catheterization, ductus arteriosus.

\section{INTRODUCCIÓN}

El término coartación de aorta, se refiere a un estrechamiento de la arteria aorta que produce una obstrucción al flujo aórtico (1). Típicamente se localiza en la aorta torácica descendente distal al origen de la arteria subclavia izquierda (llustración 1).
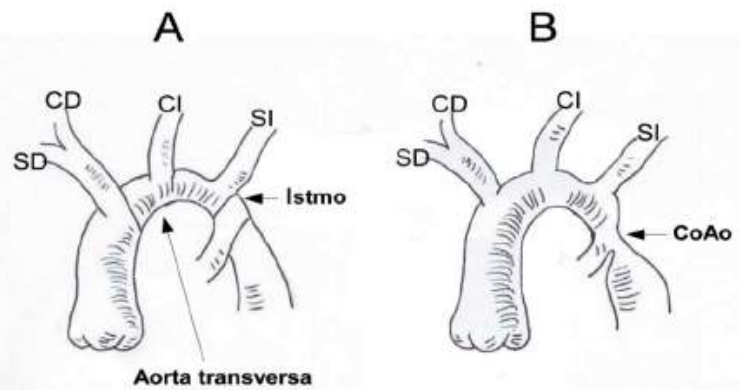

Ilustración 1. Esquema de arco aórtico: arco aórtico proximal entre el tronco braquicefálico y la Cl. Arco aórtico distal entre la Cl y la SI. Istmo aórtico entre la SI y la ampolla ductal. B: Esquema de coartación aórtica. CD: carótida derecha; Cl: carótida izquierda; CoAo: coartación aórtica A; SD: subclavia derecha; SI: subclavia izquierda. Fuente: Tomado de Ruth Solano y Luis García. Coartación de aorta e interrupción del arco aórtico. Servicio de cardiología pediátrica. Hospital Universitario La Paz, M adrid.

La mayoría de las coartaciones se sitúan en la zona de la pared posterior de la aorta, de forma opuesta a la inserción del ductus. Se suelen denominar yuxtaductales, habiendo quedado un poco en desuso los términos de coartación preductal y posductal (1).

La obstrucción se produce por una hipertrofia de la capa media de la porción posterior del vaso que protruye hacia el interior y reduce su luz; además, se ha comprobado que en las zonas adyacentes a la coartación existe tejido ductal que tiende a fibrosarse (1). 
Prácticamente todos los casos de coartación de aorta diagnosticados en el recién nacido tienen hipoplasia del istmo aórtico, y algunos, además, hipoplasia de la aorta transversa (llustración 2). Esto es debido a que la zona del istmo es, de por sí, la zona más estrecha al nacimiento. Por la fisiología de la circulación fetal, el ductus maneja gran cantidad de volumen sanguíneo con cortocircuito derecha-izquierda hacia la aorta descendente y en el istmo aórtico el flujo queda restringido.
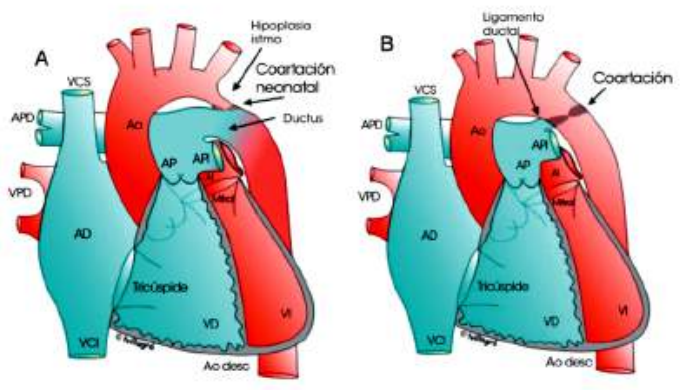

Ilustración 2. Formas principales de coartación aórtica. A: Coartación neonatal, estenosis muy severa en la zona opuesta al ductus arterioso, acompañada frecuentemente de hipoplasia a nivel del istmo y cortocircuito derecha-izquierda a nivel ductal. B: Coartación diagnosticada fuera del periodo neonatal, con estrechamiento circunscrito por debajo de la salida de la subclavia izquierda. AD: aurícula derecha; Al: aurícula izquierda; Ao: aorta; Ao des: aorta descendente; AP: arteria pulmonar, APD: arteria pulmonar derecha, API: arteria pulmonar izquierda; $\mathrm{VCl}$ : vena cava inferior, VCS: vena cava superior, VPD: venas pulmonares derechas. Fuente: tomado de F. Villagrá. www.cardiopatiascongenitas.net.

Durante la adolescencia $\mathrm{y} / 0$ edad adulta de los pacientes no intervenidos, se hace patente el desarrollo de una red de vasos colaterales, que van por la parte anterior y posterior del cuerpo: desde las arterias mamarias internas a las arterias iliacas externas, a través de las venas epigástricas, 0 bien, de las arterias tirocervicales a la aorta descendente, por las arterias intercostales, respectivamente. Este hallazgo no suele estar presente en los primeros años de vida (1).

De forma infrecuente la coartación se localiza en la aorta abdominal $(1,2)$ (Ilustración 3 ) o se presenta como un segmento de amplia longitud en la aorta descendente. Estos hallazgos se consideran entidades diferenciadas con distintos orígenes embriológicos y requieren un tratamiento quirúrgico específico.

La lesión acompañante más frecuentemente encontrada en los casos de coartación aórtica es la válvula aórtica bicúspide, descrita en una proporción situada entre el 30 y el $85 \%$ de los pacientes (3)
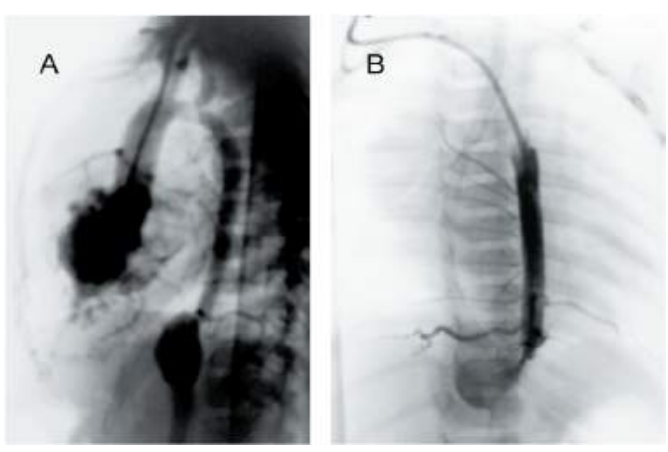

Ilustración 3. Coartación aórtica de localización atípica: coartación a nivel abdominal con importante aneurisma post-estenosis. A: visión lateral. B: Visión anteroposterior. Fuente: Tomado de Ruth Solano y Luis García. Coartación de aorta e interrupción del arco aórtico. Servicio de cardiología pediátrica. Hospital Universitario La Paz, Madrid.

Un 4-5\% de los casos tienen arteria subclavia derecha anómala, que nace por debajo de la coartación. Asimismo, también se puede asociar con malformaciones intracardiacas múltiples. En este caso, hablaríamos de coartaciones aórticas complejas (hasta un $48 \%$ del total) sobre todo cuando existe hipoplasia de algún segmento del arco aórtico. Por ejemplo, se objetiva comunicación interventricular (CIV) en 1/3 de los pacientes y/o estenosis aórtica/subaórtica en un $25 \%$. En otras ocasiones se describen lesiones obstructivas izquierdas en serie con hipoplasia de ventrículo izquierdo (VI) y alteración del aparato valvular mitral (síndrome de Shone), sin olvidar todo tipo de malformaciones cardiacas complejas (transposición de las grandes arterias, ventrículo derecho (VD) de doble salida, ventrículo único) $(1,3)$.

Debemos recordar que en torno a un $30 \%$ de las pacientes con síndrome de Turner $(1,3)$ pueden tener esta patología. Además, los pacientes con síndrome de Williams- Beuren, debido a una anormal producción de elastina, pueden presentar alteraciones en cualquier segmento aórtico (4).

\section{HISTORIA}

La coartación de la aorta fue descrita separadamente por Morgagni y Meckel en 1970, como una obstrucción localizada en la aorta cerca del ligamento arterioso. Los hallazgos clínicos fueron descritos por Wernicke en 1875 y Maude Abbot en 1828. El primer tratamiento quirúrgico fue realizado por Crafoord y Nylin en 1944 y Kirklin en 1952 (5, 6). Posteriormente múltiples publicaciones han propuesto clasificaciones clínicas, morfológicas y embriológicas. 


\section{EPIDEM IOLOGÍA}

La coartación de aorta supone aproximadamente el $5,1 \%(3-10 \%)$ de las cardiopatías congénitas y constituye la octava malformación cardiaca por orden de frecuencia. Su prevalencia se estima en 2,09 por cada 10.000 recién nacidos vivos (7). Su incidencia es mayor en varones (relación 2:1).

La mayoría de los casos aparecen de forma aislada y responden al patrón de herencia multifactorial descrito para la mayoría de las cardiopatías congénitas, pero también se han descrito casos familiares con herencia mendeliana (8). Con un hijo afecto existe un riesgo de recurrencia en los siguientes embarazos del $2 \%$, y, si hay 2 familiares con coartación, sube al $6 \%$. Si la madre tiene esta patología, el riesgo de que el feto también la desarrolle es del $4 \%$, y en el caso de que sea el padre quien la padezca, es del $2 \%$ (8).

\section{MORFOGÉNESIS}

Tres hipótesis tratan de explicar la génesis de la coartación de aorta:

1. El efecto del flujo sanguíneo in útero basado en que el istmo aórtico recibe menos flujo que la aorta ascendente, transversa y descendente, gran parte del flujo eyectado por el VI sale por los vasos del cayado antes del istmo y el flujo proveniente del VD se orienta a la aorta descendente vía conducto arterioso, razón por la cual en el RN el arco aórtico distal y el istmo son de menor calibre que la aorta ascendente y aorta descendente. La cantidad de flujo a través del arco distal y del istmo puede ser un factor importante en el desarrollo de la coartación (9).

2. Existen dos factores embriológicos: uno de estos factores es el subdesarrollo o hipoplasia del arco aórtico o del istmo. Si este factor está presente predominará una hipoplasia tubular con discreta coartación (10). El otro factor es la presencia de tejido ductal ectópico en la aorta a nivel de la inserción aórtica del conducto (10).

Esta sola anomalía puede producir coartación aórtica al crecer el tejido ductal ectópico dentro de la luz aórtica, al nivel señalado constriñe la luz aórtica después del nacimiento. Si ambos mecanismos están activos, se producirá una discreta coartación e hipoplasia tubular proximal. Parece que el tejido ductal ectópico en la aorta se presenta solamente cuando el flujo ductal fetal es de dirección normal (de derecha a izquierda) $(10,11)$.
La teoría embriológica sostiene la etiología del subdesarrollo o hipoplasia del arco aórtico o istmo provocado por lesiones cardiacas proximales que limitan el flujo anterógrado dentro de la aorta ascendente in útero $(10,11)$. La cantidad de flujo a través del arco aórtico distal y del istmo puede ser un importante determinante del crecimiento de estas estructuras vasculares. Las lesiones proximales sea una obstrucción en el lado izquierdo del corazón (12), o una inadecuada patencia del foramen oval, 0 un defecto interventricular (13), pueden estimular que el ventrículo izquierdo eyecte a través del defecto en lugar de hacerlo a través de la aorta. Puede suceder una combinación de estos factores. Como resultado el balance del gasto cardiaco fetal se altera.

Un mayor porcentaje del gasto cardiaco pasa a través del VD incrementando el flujo a través del conducto arterioso fetal patente, un menor flujo derivado del VI pasa a través del istmo aórtico. Una cantidad variable del flujo ventricular derecho vía ductus puede suplir al lecho vascular superior del cuerpo por flujo retrógrado a través del istmo (11). En la coartación con flujo fetal normal el flujo a la parte inferior del cuerpo se hace por vía anterógrada a través del istmo ( $10 \%$ a $15 \%$ del gasto cardiaco derivado desde el $\mathrm{VI}$ ) y del conducto $(50 \%$ a $60 \%$ del gasto cardiaco derivado desde el VD).

En la forma más leve de coartación, el flujo fetal pudo haber sido normal, sin embargo, el tejido ductal ectópico puede estar presente en la aorta más allá de la inserción ductal en posición contraductal. Después del nacimiento la obstrucción ductal puede producir constricción aórtica contraductal como en la coartación tipo adulto "shelflike" a nivel del ligamento arterioso (10). Una alteración leve-moderada del flujo intrauterino con o sin tejido ductal ectópico, puede explicar formas moderadas de coartación aórtica. Una saliente contraductal puede bifurcar el flujo ductal en forma retrógrada, alcanzar la subclavia izquierda y en forma anterógrada hacia la aorta descendente (14).

El arco aórtico distal y el istmo pueden ser moderadamente hipoplásicos y la coartación puede ser discreta, resultando una moderada hipoplasia tubular en adición a una discreta coartación contraductal. Si la disrupción del patrón de flujo fetal es severa puede producirse marcada hipoplasia tubular del istmo y del arco aórtico distal. Después del nacimiento la aorta presenta un calibre inadecuado para el gasto cardiaco total de la parte inferior del cuerpo produciéndose gasto 
cardiaco ductus dependiente para el hemicuerpo inferior y colapso cardiaco si el conducto se cierra. La interrupción del arco aórtico puede representar una lesión más profunda que se inicie en un estadio más precoz. Esta teoría del desarrollo vascular relacionada al flujo es sostenida por los diferentes tipos de coartación (15).

3. Considerar factores contributorios: la alteración en la formación de los arcos aórticos, anomalías en la migración de la primera arteria segmentaria que da origen a la subclavia izquierda y anomalías en la unión de estos arcos con la aorta dorsal (16).

\section{CLASIFICACIÓN}

Existen diversas formas de clasificar la coartación de la aorta, para efectos de nuestra revisión, resaltaremos la clínica, anatomopatológica y según su complejidad.

CLíNICA. Tipo Infantil (neonato e infante) y tipo adulto. El tipo infantil es grave y exige diagnóstico y manejo inmediatos, es causa de la mayor mortalidad en esta patología $(17,18)$. El tipo adulto se diagnostica después del tercer año de vida. (llustración 4).

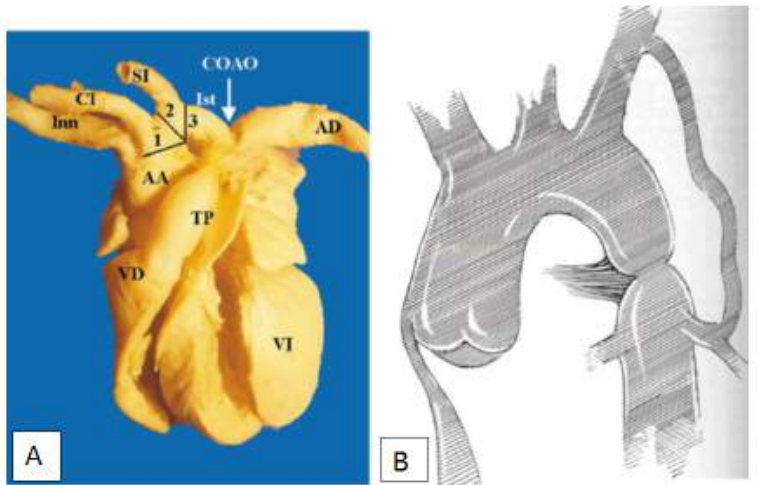

Ilustración 4. Clasificación de coartación de la aorta. A. Tipo infantil. B Tipo adulto. VD: ventrículo derecho. VI: ventrículo izquierdo. TP: tronco pulmonar. Inn: Arteria innominada. Cl: Arteria carótida izquierda. SI: Arteria subclavia izquierda. Ist: Istmo aórtico. AA: Arteria aorta. AD: Arteria aorta descendente. COAO: Coartación de la Aorta. Fuente: Tomada de Corvacho Angelica, Veliz Betty. Coartación aortica (COAO). Estudio de 55 especímenes. Revista Peruana de Cardiología Vol. XXXII № 1.

ANATOMOPATOLÓGICA. Preductal, yuxtaductal y posductal (19) según sea la localización de la coartación antes, frente o después de la unión aórtica del conducto, es frecuente la asociación de la coartación preductal con hipoplasia de una porción variable del arco aórtico, agregando severidad al cuadro. Se relacionó el tipo infantil con el preductal y el tipo adulto con el posductal, lo que no siempre es exacto (20).
SIMPLE Y COMPLEJA. En las formas simples se consideran lesiones asociadas a la coartación de la aorta: El conducto arterioso persistente, la hipoplasia del arco aórtico e istmo. Lesiones asociadas remotas son la aorta bicúspide, foramen oval permeable, CIA tipo Ostium Secundum y varios tipos de CIV $(13,21)$.

En las formas complejas la coartación aórtica se encuentra asociada a malformaciones complejas como el ventrículo izquierdo hipoplásico, anomalía de TaussigBing, transposición de los grandes vasos, atresia de la tricúspide, entre otras.

En el neonato la coartación aórtica tiene apariencia externa de continuidad entre el gran conducto arterioso y aorta descendente con hipoplasia del istmo y del arco aórtico (10). La estrechez contraductal (shelf) está presente pero es menos prominente que en el tipo adulto por la hipoplasia proximal asociada. Microscópicamente se pueden encontrar células ductales en la pared aórtica periductal (10).

\section{ANIFESTACIONESCĹNICAS \\ TIPO INFANTIL:}

La presentación clínica de la coartación aórtica es muy variada y depende de la severidad de la lesión y de su posible asociación con otras anomalías cardiacas. Aunque la forma de presentación habitual y clásica es por anomalías en la exploración física (soplo, diferencia de pulsos, hipertensión arterial) o por clínica de insuficiencia cardiaca severa en el periodo neonatal, 0 bien por medio de otros métodos diagnósticos al hallar discrepancias del tamaño de las cavidades, con un aumento del tamaño del VD respecto del izquierdo (3, 22).

El ductus arterioso juega un papel trascendental en el caso de las coartaciones aórticas severas, porque facilita el paso de flujo sanguíneo a la aorta descendente desde el $V D$, tanto en el periodo fetal como en el periodo neonatal inmediato $(3,22)$.

El ductus en el recién nacido normal se cierra en 48 horas. En los casos de coartación suele cerrarse entre los 2 y los 7 días de vida, e incluso puede permanecer permeable varias semanas con una luz muy pequeña (2). Mientras esté abierto puede no haber diferencias de pulsos entre los miembros superiores y los miembros inferiores. 
La disminución severa de calibre del ductus o el cierre del mismo produce un fallo ventricular izquierdo con congestión severa, cortocircuito izquierda-derecha por foramen oval y sobrecarga de volumen, que rápidamente desemboca en una situación de shock con dificultad respiratoria severa, acidosis y ausencia de pulsos en los miembros inferiores. Es frecuente la alteración transitoria de la función hepática y renal. Estas situaciones se hacen más patentes si hay CIV y lesiones obstructivas izquierdas asociadas.

Los casos de coartación severa se suelen manifestar en la primera o la segunda semana de vida. En la exploración física del recién nacido se puede observar palidez, frialdad acra, mala perfusión periférica, taquipnea, trabajo respiratorio, taquicardia, ritmo de galope y/o hepatomegalia. Si la situación de insuficiencia cardiaca está muy establecida, puede que no sea evidente la disminución de pulsos en los miembros inferiores, porque la tensión arterial será baja en todo el territorio arterial. Los edemas son poco frecuentes y su presencia se relaciona con el síndrome de Turner. Se debe hacer diagnóstico diferencial con sepsis, afecciones pulmonares y otras enfermedades graves neonatales, prestando especial atención en el caso de los niños prematuros (23).

Cuando la coartación aórtica no es tan severa, y una vez cerrado el ductus, los niños de mayor edad pueden permanecer asintomáticos, detectándose únicamente anomalías en la exploración física: diferencia en la intensidad de pulsos/tensión arterial en la zona proximal y distal a la coartación, hipertensión arterial en los miembros superiores y auscultación de soplo (23).

La diferencia de pulsos entre los miembros superiores y los inferiores es la alteración clínica principal, por lo que se debe recalcar la necesidad de palparlos en toda exploración pediátrica rutinaria. En algunas circunstancias, puede ser complicado, especialmente en niños obesos o con displasia de caderas. Es importante fijarse tanto en los pulsos de los dos brazos como en los carotídeos, ya que, como se ha dicho, puede haber coartaciones de aorta que se asocien con nacimiento anómalo de la arteria subclavia derecha (pulsos de menor intensidad en el brazo derecho y las piernas en comparación con el brazo izquierdo) o con origen de la subclavia izquierda distal a la coartación (pulsos de menor intensidad en el brazo izquierdo y las piernas en comparación con el brazo derecho). Cuando se advierte una diferencia de pulsos es preciso tomar la tensión arterial en los cuatro miembros con aparatos fiables de medida y manguitos adecuados al tamaño del paciente. Las diferencias de presión superiores a $20 \mathrm{mmHg}$ son significativas. En los casos más severos, el gradiente tensional puede ser superior a $70 \mathrm{mmHg}$, y la presión arterial sistólica puede llegar a $200 \mathrm{mmHg}$ en las extremidades superiores (23).

La mayoría de los niños con coartación aórtica tiene alteraciones en la auscultación y presentan soplos sistólicos precordiales de baja intensidad, que también son perceptibles en la región interescapular. Puede auscultarse clic protosistólico si se asocia a válvula aórtica bicúspide. Además, podría acompañarse de soplo sistólico de localización preesternal correspondiente a la presencia de CIV o, incluso, de soplo eyectivo por lesión obstructiva a nivel valvular/ subvalvular aórtico (23).

\section{TIPO ADULTO}

La mayoría de los niños y adultos jóvenes con sólo una discreta coartación suelen ser asintomáticos. Los principales síntomas son cefaleas, epistaxis, extremidades frías y claudicación al ejercicio; la atención debe estar dirigida al sistema cardiovascular, buscando la presencia de soplo cardíaco o hipertensión en extremidades superiores, en ausencia o una marcada disminución de los pulsos femorales, que son detectados en el examen físico (24).

En un paciente joven la manifestación más frecuente es la HTA, adicional a los hallazgos mencionados al examen físico. Es importante tener presente el riesgo de estos pacientes de presentar muerte súbita por ruptura de arterias del polígono cerebral o como consecuencia de la repercusión de la coartación sobre el ventrículo izquierdo que conduce hipertrofia, luego disfunción y posteriormente a insuficiencia cardíaca (24).

\section{MÉTODOSDIAGNÓSTICOS \\ RADIOGRAFÍA DE TÓRAX. La radiografía de tórax en el recién nacido con coartación severa muestra cardiomegalia moderada o severa acompañada de signos de hiperflujo y congestión pulmonar. Los niños mayores presentan radiografías normales 0 con cardiomegalia leve. En pacientes no intervenidos con coartación de larga evolución se pueden notar "muescas costales", que corresponden a escotaduras en el margen inferior del tercio medio de las costillas, producida por la dilatación de las arterias intercostales (23).}


ELECTROCARDIOGRAMA. En el recién nacido suele mostrar el predominio habitual del VD, y en los casos más graves, hipertrofia ventricular derecha. En niños mayores y adultos el ECG es normal, y en los casos severos aparecen signos de hipertrofia ventricular izquierda (23).

ECOCARDIOGRAFÍA. La ecocardiografía bidimensional y la ecocardiografía Doppler son el método diagnóstico fundamental para la coartación aórtica (22). La detección de la zona afecta es más fácil en recién nacidos y lactantes que en niños mayores y adolescentes, porque existe mejor ventana ecográfica. Desde planos supraesternales se puede observar una escotadura en la pared posterior de la aorta torácica y realizar mediciones del calibre de la aorta ascendente, la aorta transversa, el istmo aórtico y la aorta descendente.

Por medio del Doppler y del Doppler color estudiamos la aceleración del flujo en la zona de la coartación, calculando la diferencia de presión entre la aorta proximal y la distal a la obstrucción y, por tanto, evaluando su gravedad. Las obstrucciones severas muestran un patrón de flujo característico con prolongación diastólica (25).

\section{RESONANCIA MAGNÉTICA Y TOMOGRAFÍA} COMPUTARIZADA. La angiorresonancia magnética (angio-RM) con inyección de gadolinio por vía periférica permite hacer una evaluación detallada de la aorta mediante una reconstrucción tridimensional que muestra claramente sus defectos. Además, a diferencia de la angiografía clásica, con una sola administración de contraste se consigue la visión del arco desde múltiples planos. La limitación fundamental de la angio-RM es que es una técnica que exige la colaboración del paciente, que ha de permanecer inmóvil durante un periodo de tiempo no inferior a 15 minutos. Por ello, los niños más pequeños (generalmente $<6$ años) requieren sedación y la presencia de un anestesista (23).

La TC multicorte reduce de manera importante la duración del estudio y también permite la visualización tridimensional del arco desde múltiples planos, pero como inconveniente tiene la emisión de radiación. Para valorar el arco aórtico y sus ramas generalmente no se precisa sincronismo respiratorio (26), salvo que se quiera estudiar la válvula aórtica.

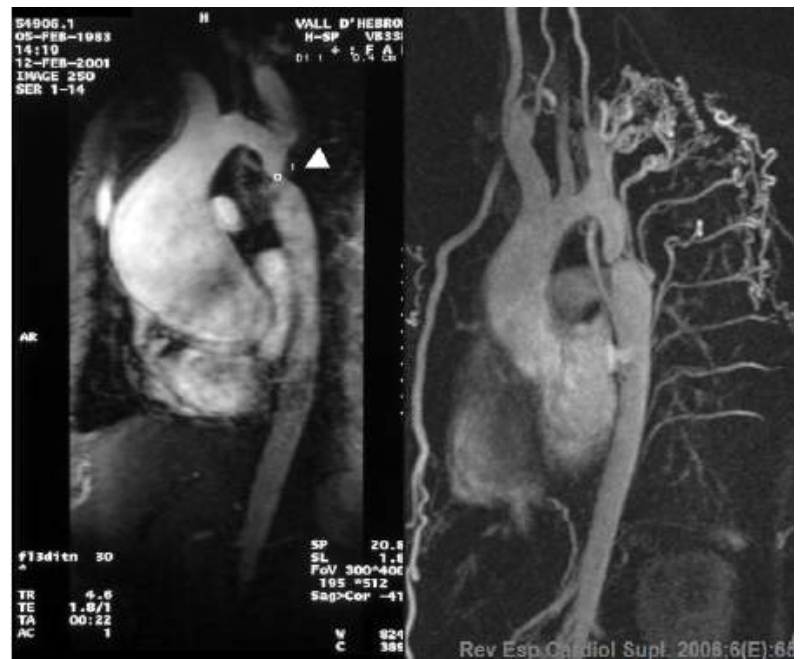

Ilustración 5: Izquierda: resonancia magnética en proyección sagital que evidencia la presencia de una coartación de aorta (flecha) y de una severa dilatación de la aorta ascendente en un paciente con válvula aórtica bicúspide. Derecha: importante circulación colateral en una coartación aórtica severa. Fuente: Tomada de Rev Esp Cardiol Supl. 2006;6(E):65-74. - Vol. 6 Núm.Supl.E DOI: 10.1157/13092061.

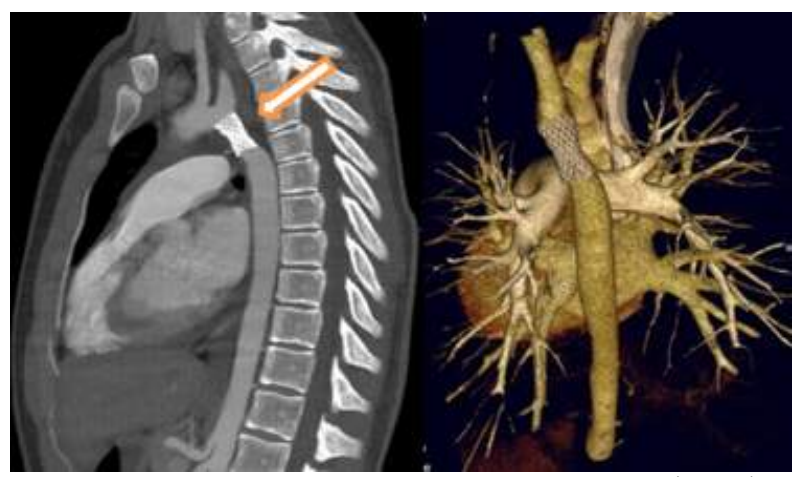

Ilustración 6. Coartación de aorta tratada con un stent (Flecha) proyección MIP (Izquierda) y reconstrucción VRT-3D (Derecha). Fuente: Tomada de Resonancia Magnética y TAC en las cardiopatías congénitas.http://www.cardiopatiascongenitas.net/pinta_htmlbd_n_p rocesocard_3.htm.

CATETERISMO CARDÍACO. Con la mejora de otras técnicas diagnósticas incruentas, el cateterismo cardiaco y la angiografía han quedado relegadas prácticamente al ámbito terapéutico. Mediante su empleo, no sólo se demuestra la localización y extensión de la zona coartada, así como la presencia de lesiones asociadas y/o de circulación colateral; sino que se adquiere conocimiento fiable de la severidad y repercusión hemodinámica de la misma (cálculo de gradientes de presión, gasto cardiaco y estimación de cortocircuitos). Los gradientes obstructivos superiores a $20 \mathrm{mmHg}$ son significativos. El cateterismo, no obstante, tiene sus limitaciones: además de la radiación emitida, el paso de un catéter por zonas de obstrucción severa es complejo, 
y se pueden presentar complicaciones en pacientes hemodinámicamente comprometidos, especialmente los recién nacidos. No hay que olvidar que en situación de insuficiencia cardiaca severa se pueden encontrar gradientes tensionales falsamente leves y que, si el ductus está patente, puede dificultar la correcta evaluación de la lesión $(3,27)$.

\section{TRATAM IENTO}

MÉDICO. El recién nacido con coartación severa puede estar en situación clínica muy grave y requerir, en primer lugar, estabilización hemodinámica mediante perfusión inmediata de PGE1 para reabrir el ductus, drogas vasoactivas y asistencia respiratoria, así como fármacos para la corrección rápida de la acidosis y del equilibrio hidroelectrolítico $(1,3)$.

La dosificación de PGE1 habitual es de 0,1 $\mu \mathrm{g} / \mathrm{kg} / \mathrm{min}$ durante 20 minutos seguida de perfusión de mantenimiento a $0,03 \mu \mathrm{g} / \mathrm{kg} / \mathrm{min}$. Para pacientes de más de 10-15 días de edad, la utilidad de la PGE1 es baja, pero ocasionalmente se ha demostrado eficaz hasta las 4-6 semanas de vida (3). Con la repermeabilización ductal aumentará la presión arterial en los miembros inferiores y mejorará el estado general, la diuresis y la perfusión periférica (3).

La hipertensión arterial del niño mayor no intervenido generalmente no responde a los fármacos hipotensores hasta después de la cirugía. Si el paciente está asintomático y su tensión arterial no supera el percentil 95 para su edad, se podrá intervenir de manera electiva (23).

QUIRÚRGICO. La coartación de aorta se puede resolver quirúrgicamente 0 a través de procedimientos de intervencionismo percutáneo (28).

En cirugía, el abordaje habitual es la toracotomía lateral izquierda. Implica el clampaje de la aorta por encima y por debajo de la obstrucción, de modo que el flujo hacia la parte inferior del cuerpo llega sólo por los vasos colaterales (29). Cuando el arco es hipoplásico o cuando se reparan simultáneamente otras lesiones asociadas, se utiliza la esternotomía media con bypass cardiopulmonar (30).

La técnica más extendida para neonatos con hipoplasia de la aorta transversa (29) es la anastomosis término-terminal ampliada $(31,32)$, que incluye la resección de la coartación y del tejido ductal con reconstrucción del arco mediante la movilización y sutura de la aorta distal hacia la concavidad de la aorta transversa.

La corrección por medio de bypass con conductos protésicos es excepcional y se reserva para adultos con una anatomía especialmente compleja o lesiones aneurismáticas importantes que impidan la sutura término-terminal $(28,29)$.

Con respecto a las complicaciones postquirúrgicas en el periodo inmediato tras la cirugía es muy frecuente la hipertensión arterial, que suele ser transitoria (33). También hay que vigilar el sangrado -especialmente en los pacientes mayores 0 en las reintervenciones-, la aparición de quilotórax y/o la parálisis del nervio recurrente, entre otras (23).

La incidencia de recoartación en neonatos y lactantes oscila entre un 15 y un $30 \%$. Se puede dar con cualquier técnica, pero es menor en la anastomosis términoterminal ampliada (existen series en la literatura con índices de recoartación global del $4 \%$ en un plazo medio de seguimiento de 4 años, del 2,9\% para los nacidos a término y del $30 \%$ para los pretérminos) (34).

La edad ideal del tratamiento quirúrgico electivo es controvertida. En un estudio retrospectivo (34) se sugiere que la edad de intervención con menor índice de reestenosis es el año y medio de vida $(<3 \%)$. Por otra parte, el retraso de la cirugía se asocia a una mayor incidencia de hipertensión arterial residual (en los casos operados antes de los 5 años es $<5 \%)(35,36)$.

La incidencia general de aneurismas en la zona quirúrgica general es del $9 \%(37,38)$. También se pueden desarrollar aneurismas en la aorta ascendente, sobre todo si existe obstrucción residual, válvula aórtica bicúspide e HTA.

La paraplejia se ha descrito en 1 de cada 500 operados de coartación (39) (estudio multicéntrico con 12.000 casos intervenidos). Infrecuentemente se presenta isquemia intestinal postoperatoria (síndrome postcoartectomía), por lo que se suele retrasar la introducción de la alimentación 48 horas (23).

La CIV es la lesión asociada que con mayor frecuencia precisa de reparación quirúrgica $(40,41)$. 
CATETERISMO CARDÍACO INTERVENCIONISTA. La coartación aórtica se puede tratar por medio de la angioplastia con catéter-balón. Esta técnica implica guiar dicho catéter, generalmente de forma retrógrada desde la arteria femoral, hasta la zona de la obstrucción e inflar un globo para dilatar la coartación. El tamaño del balón se puede aumentar gradualmente hasta que sea 2-4 veces el diámetro inicial de la zona estenótica, pero no se debe sobrepasar el diámetro de la aorta diafragmática. Ésta es la técnica de elección para el tratamiento de la reestenosis después de la cirugía (42).

La mortalidad de la dilatación de la recoartación postoperatoria es del $0-2 \%$, y el índice de reestenosis es del $16-28 \%$ en un seguimiento medio de 58 meses (27).

El uso de la angioplastia como método de abordaje de la coartación nativa (angioplastia primaria) es controvertido (43), especialmente en el caso de los neonatos. En un estudio aleatorizado (44) realizado en 1994 y reevaluado en 2005 con niños de entre 3 y 10 años $(n=36)$, se comprueba que con el paso del tiempo la incidencia de aneurismas en la zona dilatada es mayor en el grupo de la angioplastia con catéter-balón (35\% vs. $0 \%$ ), que la incidencia de lesiones vasculares es mayor también en los intervenidos percutáneamente y que la supervivencia libre de aneurismas/ reintervención es del $50 \%$ frente al $85 \%$ del grupo quirúrgico, con un seguimiento medio de 11 años.

En conclusión, aunque la angioplastia con catéterbalón se ha realizado con éxito incluso en lactantes y recién nacidos (45), el índice de reestenosis y de aneurismas en la zona de dilatación es más elevado (especialmente en $<3$ meses).

Se puede decir que la angioplastia primaria es un método útil y seguro para el tratamiento de la coartación para niños mayores con lesiones circunscritas. Generalmente se desaconseja su uso por debajo del año de vida. También se puede emplear en aquellos pacientes de alto riesgo por tener escasa circulación colateral (23).

Adicionalmente, se ha experimentado la utilización de stent intravasculares, sumados a la técnica de la angioplastia, que mejora los resultados inmediatos, pero es necesario contar con mayor información con relación a la evolución a largo plazo de estos procedimientos, donde la principal complicación sería la reestenosis por crecimiento intimal o la estenosis relativa que sucede con el crecimiento de los segmentos alrededor de la zona de implante del stent $(24,46,47)$.
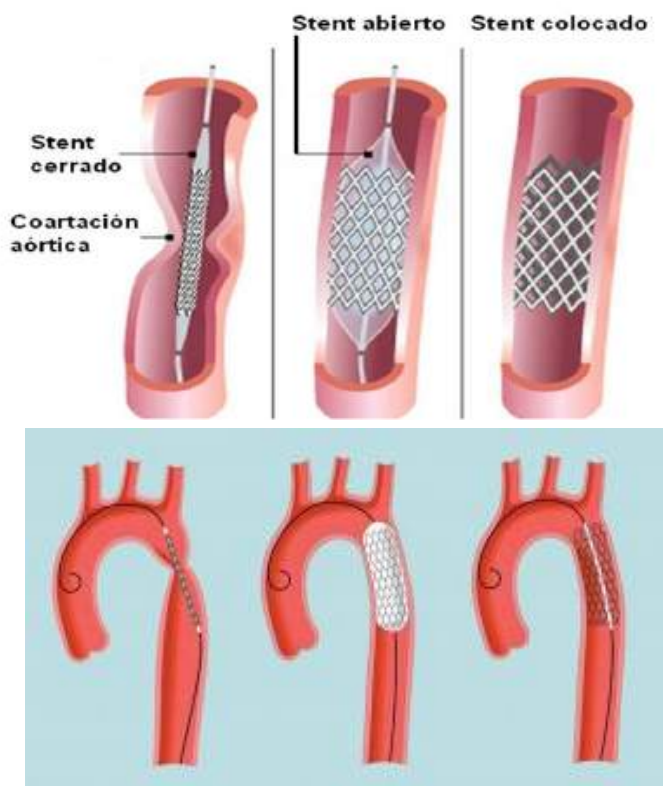

Ilustración 7. Colocación de stent para manejo de coartación de la aorta. Fuente: Tomado de http:// ductusarteriosopersisitentecoartacion.weebly.com/coartacionaortica.html.
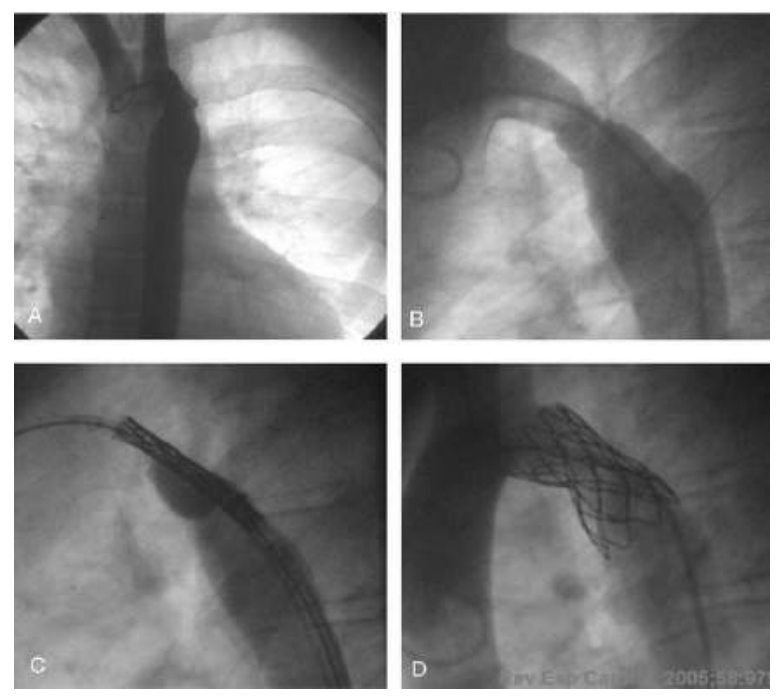

Ilustración 8. A y B: aneurisma y recoartación de aorta. C: stent posicionado en la zona de coartación de la aorta y el aneurisma (apertura de inner balloon) D: stent ya posicionado; se observa la exclusión del aneurisma y el aumento de diámetro de la zona coartada. Fuente: Tomado de Rev Esp Cardiol. 2005;58:979-83. - Vol. 58 Núm.08 DOI: $10.1157 / 13078136$.

\section{PRONÓstico}

La historia natural de la coartación aórtica aislada es mejor que la de la coartación con lesiones asociadas, pero en general tiene una alta mortalidad. Los recién nacidos con insuficiencia cardiaca (10\%) tienen muy mal 
pronóstico y una mortalidad sin intervención prácticamente total en las primeras semanas de vida. Un $20 \%$ de los pacientes morirían antes del año de vida, y el $50 \%$, antes de los 10 años $(1,3)$. Aproximadamente un $10 \%$ de los casos tendrían una clínica más leve y podrían sobrevivir sin intervención hasta la edad adulta.

En la adolescencia y en la edad adulta es muy frecuente desarrollar miocardiopatía hipertensiva, y el $90 \%$ de los pacientes no tratados fallece antes de los 50 años, siendo la edad media de muerte 35 años (48). Las causas de muerte más frecuente son la insuficiencia cardiaca (26\%), la ruptura aórtica (21\%), la endarteritis bacteriana (18\%) y la hemorragia intracraneal $(12 \%)$ (48). En los casos de coartación aórtica aislada existe una tasa de mortalidad quirúrgica inferior al $2 \%$ y una supervivencia $>95 \%$ al año y $>90 \%$ a los 5 y 10 años (34, 49).

A corto plazo, tras la intervención la mayoría de los pacientes tienen una tensión arterial normal, pero se ha descrito que un tercio de los pacientes desarrollan hipertensión de causa multifactorial en estudios de larga evolución (29). También hay evidencia de que existe una vasculopatía generalizada, en base al diferente comportamiento de los territorios vasculares periféricos pre y post-coartación (aparición de gradientes e hipertensión con el ejercicio estando en situación basal normal), por la disminución de la pulsatilidad de la aorta postestenótica y el mayor grosor intimal de la arteria carótida en relación con la arteria femoral en pacientes adultos intervenidos (23).

La hipertensión, la dilatación/disección y la rotura aórtica son riesgos que permanecen durante toda la vida del paciente y que requieren una estrecha supervisión (23). La valoración post-intervención va encaminada a determinar si hay obstrucción y/ o hipertensión arterial residual y/o aneurismas de la zona intervenida. Se considera un resultado satisfactorio cuando el gradiente residual es menor de $20-30 \mathrm{mmHg}$ sin prolongación del flujo diastólico, con tensión arterial normal y sin imágenes de dilatación (23).

Las revisiones deben incluir la toma de tensión arterial por métodos fiables, ecocardiograma (valorar también el tamaño de la aorta ascendente, la función valvular aórtica y el tamaño del VI) y radiografía de tórax, que se deben realizar a los 3, 6, 12 meses $y$, posterio rmente, cada 2 años (50).
La RM se emplea cuando hay sospecha de recoartación o aneurisma y cuando la ventana ecográfica no es la adecuada. A todos los pacientes adolescentes 0 adultos jóvenes operados en la infancia se les debe realizar RM , y también antes de planificar un embarazo (29).

El cateterismo no se emplea con fines diagnósticos sino terapéuticos durante el seguimiento, fundamentalmente para llevar a cabo la dilatación con catéter-balón en caso de recoartación aórtica. Se debe valorar cuál es el mejor momento para la realización de la angioplastia, evitando su uso precoz tras la cirugía (23).

\section{REFERENCIAS BIBLIOGRÁFICA}

1. M orris MJH M DCotaaiaaEGA, Jr BJ, Fisher DJ, Neish SR (eds.). The Science and Practice of Pediatric Cardiology. 2nd, 1347-83. eBWaWp.

2. Riemenschneider TA, Emmanouilides GC, Hirose F, Linde LM. Coarctation of the abdominal aorta in children: report of three cases and review of the literature. Pediatrics. 1969;44(5):716-26. Epub 1969/11/01. PubM ed PM ID: 5374980.

3. AM. R. Aortic arch obstruction. En: Congenital Diseases of the Heart. Clinical-Physiological considerations. Futura. Armonk NY; 2001.

4. RV L. Dysmorphology and genetics. En: Keane JF, Lock JE, Fyler DC (eds.). Nadas' Pediatric Cardiology. 2nd ed. Saunders Elsevier; 2006. p. 49-72

5. Crafoord C NG. Congenital coarctation of the aorta and its surgical treatment J Thorac Surg 1950; 37: 46.

6. Kirklin JW, Burchell HB, Pugh DG, Burke EC, Mills SD. Surgical treatment of coarctation of the aorta in a ten week old infant: report of a case. Circulation. 1952;6(3):411-4. Epub 1952/09/01. PubMed PMID: 14954538.

7. RH. B. Coarctation of the aorta. En: Emmanoulides G, Rienmenschneider $\mathrm{T}$, Allen $H$, Gutgesell $H$ (eds.). Moss and Adams. Heart Disease in Infants, Children and Adolescents. Including the Fetus and Young Adult. 5th ed. Baltimore: Williams and Wilkins; 1995. p. 1111-33.

8. Stoll C, Alembik Y, Dott B. Familial coarctation of the aorta in three generations. Annales de genetique. 1999;42(3):174-6. Epub 1999/10/20. PubMed PMID: 10526662.

9. Corvacho Angelica VB. Coartación aortica (COAO). Estudio de 55 especímenes. Revista Peruana de Cardiología Vol. XXXII № 1.

10. Ho SY, Anderson RH. Coarctation, tubular hypoplasia, 
and the ductus arteriosus. Histological study of 35 specimens. British heart journal. 1979;41(3):268-74. Epub 1979/03/01. PubMed PM ID: 426975; PubMed Central PM CID: PM CPM C482025.

11. Castañeda AR JR, Mayer J, Hanley F. . Aortic coarctatiom. In: Cardiac surgery. of the neonate and infant. Castañeda AR, Jonas RA, Mayer J, Hanley F. (ed). Saunders Company, 1994; 333-352.

12. Tawes RL, Jr., Berry CL, Aberdeen E. Congenital bicuspid aortic valves associated with coarctation of the aorta in children. British heart journal. 1969;31(1):127-8. Epub 1969/01/01. PubMed PMID: 5764958; PubM ed Central PM CID: PM CPM C487457.

13. Anderson RH, Lenox CC, Zuberbuhler JR. M orphology of ventricular septal defect associated with coarctation of aorta. British heart journal. 1983;50(2):176-81. Epub 1983/08/01. PubMed PMID: 6882605; PubMed Central PMCID: PMCPM C481392.

14. Hutchins GM . Coarctation of the aorta explained as a branch-point of the ductus arteriosus. The American journal of pathology. 1971;63(2):203-14. Epub 1971/05/01. PubMed PMID: 5090637; PubMed Central PM CID: PM CPM C2047500.

15. Rudolph AM, Heymann MA, Spitznas U. Hemodynamic considerations in the development of narrowing of the aorta. The American journal of cardiology. 1972;30(5):514-25. Epub 1972/10/01. PubM ed PM ID: 4672503.

16. Díaz G JG. Coartación de la aorta. In: G Diaz Góngora, N Sandoval Reyes, JF Vélez M oreno, G Carrillo Ángel. Cardiología Pediátrica. Ed Bogota Colombia. (Ed) Enma Ariza Herrera. 2003; 23:315-331.

17. Olley PM MN. Coarctation of the aorta in: Neonatal heart disease. Freedom RM, Benson LN, Smallhorn JF (ed). London: Springer Verlag. 1992: 376-3809.

18. Harlan JL, Doty DB, Brandt B, 3rd, Ehrenhaft JL. Coarctation of the aorta in infants. The Journal of thoracic and cardiovascular surgery. 1984;88(6):1012-9. Epub 1984/12/01. PubMed PM ID: 6503313.

19. Glancy DL, Morrow AG, Simon AL, Roberts WC. Juxtaductal aortic coarctation. Analysis of 84 patients studied hemodynamically, angiographically, and morphologically after age 1 year. The American journal of cardiology. 1983;51(3):537-51. Epub 1983/02/01. PubMed PM ID: 6218747.

20.JD. K. Coarctation of the aorta, in: Heart disease in infancy and childhood. Keith JFD, Rowe RD, Vlad P (ed). New York: Mc Millan Publishing Co, Inc, 1978; 737-760.
21. Morrow WR, Huhta JC, Murphy DJ, Jr., McNamara DG. Quantitative morphology of the aortic arch in neonatal coarctation. Journal of the American College of Cardiology. 1986;8(3):616-20. Epub 1986/09/01. PubM ed PM ID: 3745707.

22. Mareck J FM, Khambadkone S. . Aortic arch abnormalities: coarctation of the aorta and interrupted aortic arch. En: Lai WW, Mertens LL, Cohen MS and Geva T. Echocardiography in pediatric and Congenital Heart Disease: From Fetus to Adult. New Jersey: Wiley-Blackwell; 2009. p. 339-62.

23. Ruth Solana Gracia LGG. Coartación de aorta e interrupción del arco aórtico. Servicio de Cardiología Pediátrica Hospital Universitario La Paz Madrid.Capitulo 22:293-316.

24.C. Roldán Suárez OGV, L. Fernández López, L. Guerrero Llamas, J. Segura de la Morena y C. Campo Sien. Coartación de aorta del adulto. Unidad de Hipertensión. Hospital 12 de Octubre. Madrid. HIPERTENSIÓN. 2001;VOL. 18(NÚM. 5).

25. Carvalho JS, Redington AN, Shinebourne EA, Rigby ML, Gibson D. Continuous wave Doppler echocardiography and coarctation of the aorta: gradients and flow patterns in the assessment of severity. British heart journal. 1990;64(2):133-7. Epub 1990/08/01. PubM ed PM ID: 2393611; PubMed Central PM CID: PM CPM C1024353.

26. Shih MC, Tholpady A, Kramer CM, Sydnor MK, Hagspiel KD. Surgical and endovascular repair of aortic coarctation: normal findings and appearance of complications on $\mathrm{CT}$ angiography and $\mathrm{MR}$ angiography. AJR American journal of roentgenology. 2006;187(3):W302-12. Epub 2006/08/25. doi: 10.2214/ajr.05.0424. PubMed PMID: 16928909.

27. Villalba Nogales J, Fernandez-Pineda L, Herraiz Sarachaga JI, Bermudez-Canete Fernandez R, Maitre Azcarate MJ, Quero Jimenez M. [Non-surgical treatment of aortic coarctation and recoarctation]. Anales de pediatria (Barcelona, Spain : 2003). 2004;60(6):537-43. Epub 2004/06/23. PubMed PM ID: 15207165.

28. Kouchoukos NT BE, Doty DB, Hanley FL, Karp RB. . Coarctation of the aorta and interrupted aortic arch. En: Kirklin JW, Barratt-Boyes BG (eds.). Cardiac Surgery. 3rd ed. Philadelphia: Churchill Livingstone; 2003. p. 1263-327.

29. Rosenthal E. Coarctation of the aorta from fetus to adult: curable condition or life long disease process? Heart (British Cardiac Society). 2005;91(11):1495502. Epub 2005/10/19. doi: 10.1136/hrt.2004.057182. PubM ed PM ID: 16230458; 
PubM ed Central PM CID: PM CPM C1769162.

30. Kaushal S, Backer CL, Patel JN, Patel SK, Walker BL, Weigel TJ, et al. Coarctation of the aorta: midterm outcomes of resection with extended end-to-end anastomosis. The Annals of thoracic surgery. 2009;88(6):1932-8. Epub 2009/11/26. doi: 10.1016/j.athoracsur.2009.08.035. PubMed PMID: 19932265.

31. Lansman S, Shapiro AJ, Schiller MS, Ritter S, Cooper R, Galla JD, et al. Extended aortic arch anastomosis for repair of coarctation in infancy. Circulation. 1986;74(3 Pt 2):137-41. Epub 1986/09/01. PubMed PMID: 3527471.

32. Bigdelian $\mathrm{H}$, Sedighi M. Repair of aortic coarctation in infancy: A 10-year clinical experience. Asian cardiovascular \& thoracic annals. 2016. Epub 2016/04/17. doi: 10.1177/0218492316643841. PubM ed PM ID: 27084872.

33. O'Sullivan JJ, Derrick G, Darnell R. Prevalence of hypertension in children after early repair of coarctation of the aorta: a cohort study using casual and 24 hour blood pressure measurement. Heart (British Cardiac Society). 2002;88(2):163-6. Epub 2002/07/16. PubMed PMID: 12117846; PubMed Central PM CID: PM CPM C1767207.

34. Thomson JD, Mulpur A, Guerrero R, Nagy Z, Gibbs JL, Watterson KG. Outcome after extended arch repair for aortic coarctation. Heart (British Cardiac Society). 2006;92(1):90-4. Epub 2005/04/23. doi: 10.1136/hrt.2004.058685. PubMed PM ID: 15845612; PubM ed Central PM CID: PM CPM C1860999.

35. Younoszai AK, Reddy VM, Hanley FL, Brook MM. Intermediate term follow-up of the end-to-side aortic anastomosis for coarctation of the aorta. The Annals of thoracic surgery. 2002;74(5):1631-4. Epub 2002/11/21. PubM ed PM ID: 12440621.

36. Dodge-Khatami A, Backer CL, Mavroudis C. Risk factors for recoarctation and results of reoperation: a 40-year review. Journal of cardiac surgery. 2000;15(6):369-77. Epub 2001/10/27. PubMed PMID: 11678458.

37. Maxey TS, Serfontein SJ, Reece TB, Rheuban KS, Kron IL. Transverse arch hypoplasia may predispose patients to aneurysm formation after patch repair of aortic coarctation. The Annals of thoracic surgery. 2003;76(4):1090-3. Epub 2003/10/08. PubMed PMID: 14529992.

38. von Kodolitsch $Y$, Aydin MA, Koschyk DH, Loose R, Schalwat I, Karck M, et al. Predictors of aneurysmal formation after surgical correction of aortic coarctation. Journal of the American College of
Cardiology. 2002;39(4):617-24. Epub 2002/02/19. PubM ed PMID: 11849860.

39. Brewer LA, 3rd, Fosburg RG, Mulder GA, Verska JJ. Spinal cord complications following surgery for coarctation of the aorta. A study of 66 cases. The Journal of thoracic and cardiovascular surgery. 1972;64(3):368-81. Epub 1972/09/01. PubMed PM ID: 5054875.

40. Gaynor JW. Management strategies for infants with coarctation and an associated ventricular septal defect. The Journal of thoracic and cardiovascular surgery. 2001;122(3):424-6. Epub 2001/09/08. doi: 10.1067/mtc.2001.116942. PubMed PMID: 11547289.

41.Gaynor JW, Wernovsky G, Rychik J, Rome JJ, DeCampli WM, Spray TL. Outcome following singlestage repair of coarctation with ventricular septal defect. European journal of cardio-thoracic surgery : official journal of the European Association for Cardio-thoracic Surgery. 2000;18(1):62-7. Epub 2000/06/28. PubM ed PM ID: 10869942.

42. Siblini G, Rao PS, Nouri S, Ferdman B, Jureidini SB, Wilson $A D$. Long-term follow-up results of balloon angioplasty of postoperative aortic recoarctation. The American journal of cardiology. 1998;81(1):61-7. Epub 1998/02/14. PubM ed PM ID: 9462608.

43. Patel HT, Madani A, Paris YM, Warner KG, Hijazi ZM . Balloon angioplasty of native coarctation of the aorta in infants and neonates: is it worth the hassle? Pediatric cardiology. 2001;22(1):53-7. Epub 2000/12/21. doi: 10.1007/s002460010153. PubMed PM ID: 11123129.

44. Cowley CG, Orsmond GS, Feola P, McQuillan L, Shaddy RE. Long-term, randomized comparison of balloon angioplasty and surgery for native coarctation of the aorta in childhood. Circulation. 2005;111(25):3453-6. Epub 2005/06/16. doi: 10.1161/circulationaha.104.510198. PubMed PM ID: 15956126.

45. Benito Bartolome F. [Transluminal dilatation of critical aortic stenosis and coarctation of a newborn infant aorta]. Anales espanoles de pediatria. 2000;53(2):148-50. Epub 2000/11/21. PubMed PM ID: 11083956.

46. Suarez de Lezo J, Pan M, Romero M, Medina A, Segura J, Pavlovic D, et al. Balloon-expandable stent repair of severe coarctation of aorta. American heart journal. 1995;129(5):1002-8. Epub 1995/05/01. PubM ed PM ID: 7732957.

47. Bondanza S, Calevo M G, Marasini M. Early and LongTerm Results of Stent Implantation for Aortic 
Coarctation in Pediatric Patients Compared to Adolescents: A Single Center Experience. Cardiology research and practice. 2016;2016:4818307. Epub 2016/03/01. doi: 10.1155/2016/4818307. PubMed PMID: 26925287; PubMed Central PMCID: PM CPM C4748096.

48. Campbell M. Natural history of coarctation of the aorta. British heart journal. 1970;32(5):633-40. Epub 1970/09/01. PubMed PMID: 5470045; PubMed Central PM CID: PM CPM C487385.

49. M CElhinney DB, Yang SG, Hogarty AN, Rychik J, Gleason MM, Zachary $\mathrm{CH}$, et al. Recurrent arch obstruction after repair of isolated coarctation of the aorta in neonates and young infants: is low weight a risk factor? The Journal of thoracic and cardiovascular surgery. 2001;122(5):883-90. Epub 2001/11/02. doi: 10.1067/mtc.2001.116316. PubM ed PM ID: 11689792.

50. Maroto C EdSF, Herráiz I, Zabala JI. . Guías de práctica clínica de la Sociedad Española de Cardiología en las cardiopatías congénitas más frecuentes. Rev. Esp Cardiol 2001; 54: 67-82.• 